\title{
Extended scaling and residual flavor symmetry in the neutrino Majorana mass matrix
}

\author{
Rome Samanta $^{1, \mathrm{a}}$, Probir Roy ${ }^{2, \mathrm{~b}}$, Ambar Ghosal ${ }^{1, \mathrm{c}}$ \\ ${ }^{1}$ Astroparticle Physics and Cosmology Division, Saha Institute of Nuclear Physics, HBNI, Kolkata 700064, India \\ ${ }^{2}$ Center for Astroparticle Physics and Space Science, Bose Institute, Kolkata 700091, India
}

Received: 26 July 2016 / Accepted: 21 November 2016 / Published online: 30 November 2016

(C) The Author(s) 2016. This article is published with open access at Springerlink.com

\begin{abstract}
The residual symmetry approach, along with a complex extension for some flavor invariance, is a powerful tool to uncover the flavor structure of the $3 \times 3$ neutrino Majorana mass matrix $M_{v}$ toward gaining insights into neutrino mixing. We utilize this to propose a complex extension of the real scaling ansatz for $M_{v}$ which was introduced some years ago. Unlike the latter, our proposal allows a nonzero mass for each of the three light neutrinos as well as a nonvanishing $\theta_{13}$. The generation of light neutrino masses via the type-I seesaw mechanism is also demonstrated. A major result of this scheme is that leptonic Dirac CP-violation must be maximal while atmospheric neutrino mixing does not need to be exactly maximal. Moreover, each of the two allowed Majorana phases, to be probed by the search for nuclear $0 \nu \beta \beta$ decay, has to be at one of its two CP-conserving values. There are other interesting consequences such as the allowed occurrence of a normal mass ordering which is not favored by the real scaling ansatz. Our predictions will be tested in ongoing and future neutrino oscillation experiments at $\mathrm{T} 2 \mathrm{~K}, \mathrm{NO} v \mathrm{~A}$ and DUNE.
\end{abstract}

\section{Introduction}

The masses and mixing properties of the three light neutrinos are beginning to get pinned down. Though the precise mass values are still unknown, upper limits on them have been pushed down to fractions of electron volts. Furthermore, it is already known that at least one of the neutrinos must be heavier than about $50 \mathrm{meV}$. Additionally, the three angles which describe their mixing have become reasonably well known with $\theta_{12} \sim 34^{\circ}, \theta_{23} \sim 45^{\circ}$ and $\theta_{13} \sim 8^{\circ}$. Understanding this mixing phenomenon (with one small and two

\footnotetext{
a e-mail: rome.samanta@saha.ac.in

b e-mail: probirrana@gmail.com

c e-mail: ambar.ghosal@saha.ac.in
}

large angles) has emerged as a major challenge. As ongoing experiments feed in more and more information on neutrino masses and mixing, the flavor structure of the $3 \times 3$ neutrino mass matrix $M_{v}$ is being slowly uncovered. Many of its features still remain unknown nonetheless and continue to intrigue theoretical investigators. (Up-to-date overviews of these issues and their investigations along with original references may be found in the two review articles quoted in Refs. $[1,2]$.) Especially tantalizing is the predicted phenomenon of leptonic $\mathrm{CP}$-violation which likely to have implications for leptogenesis [3-5]. As yet, there is no statistically reliable definitive experimental result on leptonic CPviolation. However, hints of a near-maximal CP-violation, with the phase $\delta$ being $\simeq 3 \pi / 2$, have emerged from results reported by the T2K [6], NOvA [7] and Super-Kamiokande [8] experiments. Similarly, a recent global analysis [9] of all neutrino data is hinting at a nonmaximal value of $\sin ^{2} 2 \theta_{23}$. Another yet unresolved question of great interest is that of neutrino mass ordering: normal vs. inverted. In addition, one would like to know if the three neutrinos are Majorana or Dirac particles - to be presumably determined by a future observation of nuclear $0 \nu \beta \beta$ decay [10].

Let us start with the minimal supposition that there are only three light and flavored left-chiral neutrinos and that they are Majorana in character. The neutrino mass term in the Lagrangian density now reads

$$
-\mathcal{L}_{\text {mass }}^{v}=\frac{1}{2} \bar{\nu}_{l}^{C}\left(M_{\nu}\right)_{l m} v_{m}+\text { h.c. }
$$

with $\nu_{l}^{C}=C \bar{\nu}_{l}^{T}$ and the subscripts $l, m$ spanning the lepton flavor indices $e, \mu, \tau . M_{\nu}$ is a complex symmetric matrix $\left(M_{v}^{*} \neq M_{v}=M_{v}^{T}\right)$ which can be put into a diagonal form by a similarity transformation with a unitary matrix $U$ :

$U^{T} M_{\nu} U=M_{v}^{d} \equiv \operatorname{diag}\left(m_{1}, m_{2}, m_{3}\right)$.

Here $\mathrm{m}_{i}(i=1,2,3)$ are real and positive masses. We choose to work in a weak basis [11], in which the charged lepton 
mass matrix is diagonal with real and positive elements, i.e. $M_{l}=$ diag. $\left(m_{e}, m_{\mu}, m_{\tau}\right)$ and the unphysical phases of $U$ are absorbed into the neutrino fields. Now

$$
\begin{aligned}
U & =U_{\mathrm{PMNS}} \\
& \equiv\left(\begin{array}{ccc}
c_{12} c_{13} & e^{i \frac{\alpha}{2}} s_{12} c_{13} & s_{13} e^{-i\left(\delta-\frac{\beta}{2}\right)} \\
-s_{12} c_{23}-c_{12} s_{23} s_{13} e^{i \delta} & e^{i \frac{\alpha}{2}}\left(c_{12} c_{23}-s_{12} s_{13} s_{23} e^{i \delta}\right) & c_{13} s_{23} e^{i \frac{\beta}{2}} \\
s_{12} s_{23}-c_{12} s_{13} c_{23} e^{i \delta} & e^{i \frac{\alpha}{2}}\left(-c_{12} s_{23}-s_{12} s_{13} c_{23} e^{i \delta}\right) & c_{13} c_{23} e^{i \frac{\beta}{2}}
\end{array}\right)
\end{aligned}
$$

with $c_{i j} \equiv \cos \theta_{i j}, s_{i j} \equiv \sin \theta_{i j}$ and $\theta_{i j}=[0, \pi / 2]$. CPviolation enters through nontrivial values of the Dirac phase $\delta$ and of the Majorana phases $\alpha, \beta$ with $\delta, \alpha, \beta=[0,2 \pi]$. We follow the PDG convention [12] on these angles and phases except that we denote the Majorana phases by $\alpha$ and $\beta$. In principle there could also be a phase matrix with $U_{\text {PMNS }}$ if we work in a weak basis where $M_{l}$ is diagonal but where the unphysical phases are not absorbed in the neutrino fields. It is demonstrated later that even if we include the unphysical phase matrix, our result remains the same which is obvious, since physical results are basis independent.

Quite a few different hypotheses have been advanced over several decades on the flavor structure of $M_{\nu}$, as reviewed in the first article of Refs. [1,2]. We zero in on an ansatz made some years ago $[13,14]$ that we call Simple Real Scaling (SRS). This posits the relations

$$
\frac{\left(M_{v}^{\mathrm{SRS}}\right)_{e \mu}}{\left(-M_{v}^{\mathrm{SRS}}\right)_{e \tau}}=\frac{\left(M_{\nu}^{\mathrm{SRS}}\right)_{\mu \mu}}{\left(-M_{v}^{\mathrm{SRS}}\right)_{\mu \tau}}=\frac{\left(M_{\nu}^{\mathrm{SRS}}\right)_{\tau \mu}}{\left(-M_{\nu}^{\mathrm{SRS}}\right)_{\tau \tau}}=k,
$$

where $k$ is a real and positive dimensionless scaling factor. It is straightforward to induce from (1.4) the form of the neutrino Majorana mass matrix:

$M_{v}^{\mathrm{SRS}}=\left(\begin{array}{ccc}X & -Y k & Y \\ -Y k & Z k^{2} & -Z k \\ Y & -Z k & Z\end{array}\right)$.

Here $X, Y, Z$ are complex mass dimensional quantities that are a priori unknown. We consistently denote complex (real) quantities by capital (small) letters throughout. We have chosen appropriate negative signs in (1.4) and (1.5) to be in conformity with the PDG convention [12] on the form of $U_{\text {PMNS }}$ that emerges from (1.5). It was pointed out by Mohapatra and Rodejohann $[13,14]$ that -in the basis where the charged lepton mass matrix is diagonal-(1.5) can be realized from the larger symmetry group $D_{4} \times \mathbb{Z}_{2}$. This ansatz of SRS led to a sizable body of research [15$21]$. But it predicts a vanishing $s_{13}$ (and hence no measurable leptonic Dirac CP-violation) as well as an inverted neutrino mass hierarchy (i.e. $m_{2,1}>m_{3}$ ) with $m_{3}=0$. While the latter result is still allowed within current experimental bounds, a null value of $s_{13}$ has been ruled out at more than $10 \sigma$ [22]. Thus SRS, as it stands, has to be abandoned.

We want to consider an extended version of (1.5) which allows a nonvanishing $s_{13}$. To this end, we employ the method of complex extension which in turn is based on the idea of the residual symmetry $\mathbb{Z}_{2} \times \mathbb{Z}_{2}$ [23-25] of $M_{\nu}$. This is explained in Sect. 3 below. As detailed in the subsequent Sect. 4, the complex extension (CES for Complex Extended Scaling) leads to the neutrino mass matrix

$M_{v}^{\mathrm{CES}}=\left(\begin{array}{ccc}x & -y_{1} k+i y_{2} k^{-1} & y_{1}+i y_{2} \\ -y_{1} k+i y_{2} k^{-1} & z_{1}-w k^{-1}\left(k^{2}-1\right)-i z_{2} & w-i z_{2}(2 k)^{-1}\left(k^{2}-1\right) \\ y_{1}+i y_{2} & w-i z_{2}(2 k)^{-1}\left(k^{2}-1\right) & z_{1}+i z_{2}\end{array}\right)$

Here $x, y_{1,2}, z_{1,2}$, and $w$ are real mass dimensional quantities that are a priori unknown. It will be shown that $M_{v}^{\mathrm{CES}}$ of (1.6) can accommodate a nonzero value for each of $m_{1}, m_{2}, m_{3}$ and can fit the extant data on $\Delta m_{21}^{2} \equiv$ $m_{2}^{2}-m_{1}^{2},\left|\Delta m_{32}^{2}\right| \equiv\left|m_{3}^{2}-m_{2}^{2}\right|$ as well as on $\theta_{12}$ and $\theta_{13}$. The relation $\tan \theta_{23}=k^{-1}$ is a consequence so that the presently allowed range of $\tan \theta_{23}$ around unity would yield the permitted domain of the variation of the scaling parameter $k$ to be close to 1. Furthermore, (1.6) leads to the result that $\alpha, \beta=0$ or $\pi$, i.e. there is no Majorana $\mathrm{CP}$-violation, and the verifiable/falsifiable prediction that $\cos \delta=0$, i.e. leptonic Dirac CP-violation is maximal. We have no statement on the sign of $\sin \delta$ so that $\delta$ can be either $\pi / 2$ or $3 \pi / 2$. Furthermore, we show that a normal mass ordering (with $m_{2,1}<m_{3}$ ) is allowed in addition to an inverted one $\left(m_{2,1}>m_{3}\right)$ in the parameter space of the model.

The rest of the paper is organized as follows. In Sect. 2 we elucidate the meaning of the residual $\mathbb{Z}_{2} \times \mathbb{Z}_{2}$ discrete symmetry of $M_{v}$ in terms of its invariance under two separate similarity transformations. Simple real scaling and its real generalization are discussed in Sect. 3. Section 4 contains a presentation of the procedure of complex extension; this is first illustrated for $\mu \tau$ interchange symmetry and then applied to the scaling transformation to lead to the proposed $M_{v}^{\text {CES }}$ of (1.6) as well as its main consequences, namely $\tan \theta_{23}=k^{-1}$ and $\cos \delta=0$ plus the allowed occurrence of a normal mass ordering. The origin of the neutrino mass matrix $M_{v}^{\mathrm{CES}}$ in our scheme from the type-I seesaw mechanism is shown in Sect. 5. Detailed phenomenological implications of $M_{v}^{\mathrm{CES}}$ are worked out numerically in Sect. 6 and fitted with the current data yielding various $3 \sigma$-allowed regions in the parameter space; the application of our results to forthcoming experiments on nuclear $0 \nu \beta \beta$ decay and neutrino oscillations is also discussed in the same section. Section 7 summarizes our conclusions. 


\section{Meaning of residual flavor symmetry of $M_{v}$}

It would be useful to focus on the feature [23-25] of $M_{v}$ that it has a residual (sometimes called 'remnant' [26]) $\mathbb{Z}_{2} \times \mathbb{Z}_{2}$ flavor symmetry and at the same time review the representation content of the latter. Such an exercise will enable us to set up the theoretical machinery needed to apply the idea to SRS. In addition, this will lead us to its real generalization as well as to its complex extension.

Let $G$ be a generic $3 \times 3$ unitary matrix representation of some horizontal symmetry of $M_{v}$ effected through the similarity transformation

$G^{T} M_{\nu} G=M_{\nu}$.

Equations (1.2) and (2.1) lead to the conclusion that the unitary matrix $U^{\prime} \equiv G U$ also puts $M_{v}$ into a diagonal form by a similarity transformation, i.e. $U^{\prime T} M_{\nu} U^{\prime}=M_{\nu}^{d}$. It can then be shown [23-25] that, if $m_{1}, m_{2}$ and $m_{3}$ are nondegenerate, $G$ has eigenvalues \pm 1 and is diagonalized by $U$. Thus

$$
\begin{aligned}
G U & =U d, \\
d^{2} & =I .
\end{aligned}
$$

Here $d$ is a $3 \times 3$ diagonal matrix in flavor space with $d_{l m}= \pm \delta_{l m}$. There are eight possible distinct forms for $d$. Two of these are trivial - being the unit and the negative unit matrices. Of the remaining six, three are negatives of the other three. Finally, we have three $G_{a}$ 's $(\mathrm{a}=1,2,3)$ but it is sufficient to consider any two of those as independent on account of the relation $G_{a}=\epsilon_{a b c} G_{b} G_{c}$. The two independent $G_{a}$ (chosen here as $G_{2,3}$ ) are representations of a residual $\mathbb{Z}_{2} \times \mathbb{Z}_{2}$ symmetry in the Majorana mass term of the neutrino Lagrangian. It follows from (2.2) and (2.3) that

$$
\begin{aligned}
G^{2} & =I, \\
\operatorname{det} G & = \pm 1 .
\end{aligned}
$$

The eigenvalue equation (2.2) needs to be considered for the two independent $d$ 's, i.e. $d_{2}$ and $d_{3}$, corresponding respectively to $G_{2}$ and $G_{3}$. Suppose we choose

$d_{2}=\operatorname{diag}(-1,1,-1)$,

$d_{3}=\operatorname{diag}(-1,-1,1)$,

for $\operatorname{det} G=1$. (The choice for the case $\operatorname{det} G=-1$ is a trivial extension with $-d_{2}$ and $-d_{3}$.) Now

$G_{2,3}=U d_{2,3} U^{\dagger}$

can be obtained by use of the explicit form of $U$ as given in (1.3). For instance, let us consider the situation for $\mu \tau$ interchange symmetry [27-29] which implies $\theta_{23}=\pi / 4$ and $\theta_{13}=0$. Now we obtain

$$
\begin{aligned}
G_{2} & =\left(\begin{array}{ccc}
-\cos 2 \theta_{12} & 2^{-\frac{1}{2}} \sin 2 \theta_{12} & -2^{-\frac{1}{2}} \sin 2 \theta_{12} \\
2^{-\frac{1}{2}} \sin 2 \theta_{12} & -\frac{1}{2}\left(1-\cos 2 \theta_{12}\right) & -\frac{1}{2}\left(1+\cos 2 \theta_{12}\right) \\
-2^{-\frac{1}{2}} \sin 2 \theta_{12} & -\frac{1}{2}\left(1+\cos 2 \theta_{12}\right) & -\frac{1}{2}\left(1-\cos 2 \theta_{12}\right)
\end{array}\right), \\
G_{3}^{\mu \tau} & =\left(\begin{array}{ccc}
-1 & 0 & 0 \\
0 & 0 & 1 \\
0 & 1 & 0
\end{array}\right) .
\end{aligned}
$$

The above $G_{3}$ explicitly implements $\mu \tau$ interchange in the neutrino flavor basis and hence has been labeled with the superscript $\mu \tau$. Thus one can now identify one of the two residual $\mathbb{Z}_{2} \mathrm{~s}$ as $\mathbb{Z}_{2}^{\mu \tau}$. The full residual symmetry in this case is $\mathbb{Z}_{2} \times \mathbb{Z}_{2}^{\mu \tau}$. Our aim would be to perform a similar task with scaling symmetry in obtaining a $\mathbb{Z}_{2}^{\text {scaling }}$. It may be mentioned that some authors [30] have generalized $G_{3}^{\mu \tau}$ to

$G_{3}^{G \mu \tau}=\left(\begin{array}{ccc}-1 & 0 & 0 \\ 0 & \cos 2 \theta_{23} & \sin 2 \theta_{23} \\ 0 & \sin 2 \theta_{23} & \cos 2 \theta_{23}\end{array}\right)$,

which can accommodate an arbitrary $\theta_{23}$ but still has $\theta_{13}=0$. A somewhat different use of the residual symmetry approach with another pair of $\mathbb{Z}_{2}$ was made in Refs. [31-33].

A comment on the use of the residual $\mathbb{Z}_{2} \times \mathbb{Z}_{2}$ symmetry would be in order. One could start from any arbitrary ansatz on $U_{\mathrm{PMN} S}$, reconstruct the residual $\mathbb{Z}_{2} \times \mathbb{Z}_{2}$ symmetry and work out the consequences. However, the $\mathbb{Z}_{2} \times \mathbb{Z}_{2}$ symmetry emerging from an arbitrary ansatz may not follow from a larger symmetry group or have some deeper flavor meaning. The SRS ansatz has been shown to follow $[13,14]$ from a larger flavor symmetry group $D_{4} \times \mathbb{Z}_{2}$.

\section{Simple real scaling and its real generalization}

SRS and the corresponding $M_{\nu}^{\text {SRS }}$, cf. (1.5), were already introduced in Sect. 1. It is evident from (1.5) that the latter has a vanishing determinant, i.e. one null eigenvalue. The corresponding eigenvector, given that $\theta_{12}$ and $\theta_{23}$ are known to be hugely nonzero, can be identified only with the third column of $U^{\text {SRS }}$ and written as

$C_{3}^{\mathrm{SRS}}=\left(\begin{array}{c}0 \\ \left(1+k^{2}\right)^{-\frac{1}{2}} e^{i \frac{\beta}{2}} \\ k\left(1+k^{2}\right)^{-\frac{1}{2}} e^{i \frac{\beta}{2}}\end{array}\right)$.

Two immediate consequences are that $m_{3}=0$, i.e. the neutrino mass ordering is inverted $\left(m_{2,1}>m_{3}\right)$, and $\theta_{13}=0$. The full $U^{\text {SRS }}$ can be written with an undetermined angle $\theta_{12}$ and the corresponding $c_{12}, s_{12}$ as 
$U^{\mathrm{SRS}}=\left(\begin{array}{ccc}c_{12} & s_{12} e^{i \frac{\alpha}{2}} & 0 \\ -k\left(1+k^{2}\right)^{-\frac{1}{2}} s_{12} & k\left(1+k^{2}\right)^{-\frac{1}{2}} c_{12} e^{i \frac{\alpha}{2}} & \left(1+k^{2}\right)^{-\frac{1}{2}} e^{i \frac{\beta}{2}} \\ \left(1+k^{2}\right)^{-\frac{1}{2}} s_{12} & -\left(1+k^{2}\right)^{-\frac{1}{2}} c_{12} e^{i \frac{\alpha}{2}} & k\left(1+k^{2}\right)^{-\frac{1}{2}} e^{i \frac{\beta}{2}}\end{array}\right)$.

A comparison between (1.3) and (3.2) immediately yields

$\tan \theta_{23}=k^{-1}$.

As we shall see, (3.3) is going to survive both the real generalization and the complex CP-transformed extension of SRS.

An expression for $G_{3}^{\text {scaling }}$ as a representation for $\mathbb{Z}_{2}^{\text {scaling }}$ can now be derived by use of (2.8). On utilizing $U^{\text {SRS }}$ from (3.2) and $d_{3}$ from (2.7), we have

$$
\begin{aligned}
G_{3}^{\text {scaling }} & =\left(\begin{array}{ccc}
-1 & 0 & 0 \\
0 & \left(1-k^{2}\right)\left(1+k^{2}\right)^{-1} & 2 k\left(1+k^{2}\right)^{-1} \\
0 & 2 k\left(1+k^{2}\right)^{-1} & -\left(1-k^{2}\right)\left(1+k^{2}\right)^{-1}
\end{array}\right) \\
& =\left(G_{3}^{\text {scaling }}\right)^{T} .
\end{aligned}
$$

The $\mathbb{Z}_{2}^{\text {scaling }}$ symmetry of $M_{v}^{\text {SRS }}$ ensures that

$$
\left(G_{3}^{\text {scaling }}\right)^{T} M_{v}^{\mathrm{SRS}} G_{3}^{\text {scaling }}=M_{v}^{\mathrm{SRS}} \text {. }
$$

It may be noted that (3.5) does not lead uniquely to the form (1.5). Further, while the form of $G_{3}^{\text {scaling }}$ follows uniquely from $U^{\text {SRS }}$ of (3.2) via the relation been $G_{3}$ and $d_{3}$, the reverse is not the case. Indeed, though the third column of $U$, reconstructed from $G_{3}^{\text {scaling }}$, must be $C_{3}^{\text {SRS }}$ of (3.1) since $\left(d_{3}\right)_{33}=1$, its first two columns could be an arbitrary orthogonal pair. That occurs because of the degeneracy of the $(1,1)$ and $(2,2)$ elements in $d_{3}$. The full residual symmetry of $M_{v}^{\text {SRS }}$ is $\mathbb{Z}_{2}^{k} \times \mathbb{Z}_{2}^{\text {scaling }}$, where a representation for $\mathbb{Z}_{2}^{k}$ is $G_{2}^{k}=U^{\text {SRS }} d_{2} U^{\text {SRS } \dagger}$. Explicitly,

$G_{2}^{k}=\left(\begin{array}{ccc}-\cos 2 \theta_{12} & k\left(1+k^{2}\right)^{-1} \sin 2 \theta_{12} & -\left(1+k^{2}\right)^{-1} \sin 2 \theta_{12} \\ k\left(1+k^{2}\right)^{-1} \sin 2 \theta_{12} & -k^{2}\left(1+k^{2}\right)^{-1}\left(1-\cos 2 \theta_{12}\right) & -k\left(1+k^{2}\right)^{-1}\left(1+\cos 2 \theta_{12}\right) \\ -\left(1+k^{2}\right)^{-1} \sin 2 \theta_{12} & -k\left(1+k^{2}\right)^{-1}\left(1+\cos 2 \theta_{12}\right) & -\left(1+k^{2}\right)^{-1}\left(k^{2}-\cos 2 \theta_{12}\right)\end{array}\right)$,

$\left(G_{2}^{k}\right)^{T} M_{v}^{\mathrm{SRS}} G_{2}^{k}=M_{v}^{\mathrm{SRS}}$.

Grimus and Lavoura made an alternative proposal, namely the complex-extended invariance relation

$\left(G_{3}^{\mu \tau}\right)^{T} M_{v} G_{3}^{\mu \tau}=M_{v}^{*}$.

A good check is that, for $k=1$, the scaling procedure just reduces to $\mu \tau$ interchange with the additional constraint $M_{\mu \mu}^{v}=M_{\mu \tau}^{v}$. But the point of real interest is that $M_{v}^{\text {SRS }}$ of (1.5) is not the most general form obeying (3.5). The latter may be worked out to be

$M_{v}^{\mathrm{GRS}}=\left(\begin{array}{ccc}x & -Y k & Y \\ -Y k & Z-W k^{-1}\left(k^{2}-1\right) & W \\ Y & W & Z\end{array}\right)$,

where $W$ is another a priori unknown mass dimensional complex quantity. We call this form of $M_{\nu}$ the Generalized Real
Scaling ansatz and denote it by the superscript GRS. Evidently, the specific choice $W=-Z k$ reduces $M_{v}^{\mathrm{GRS}}$ to $M_{v}^{\mathrm{SRS}}$. The neutrino mass matrix $M_{v}^{\mathrm{GRS}}$ of (3.8) has interesting properties. For one thing, it has a determinant which does not appear to vanish. Therefore, we take all neutrino masses to be nonzero and can accommodate a nonzero $m_{3}$ and in principle a normal mass ordering with $m_{2,1}<m_{3}$. However, being invariant under a similarity transformation by $G_{3}^{\text {scaling }}$ of (3.4), the third column of the corresponding $U^{\mathrm{GRS}}$ is constrained to be $C_{3}^{\mathrm{SRS}}$ of (3.1). Consequently, one obtains a vanishing $\theta_{13}$ which is now experimentally known to be nonzero. Thus $M_{v}^{\mathrm{GRS}}$ of (3.8) is unacceptable. A more extended version of scaling in the neutrino mass matrix is needed to describe nature. This is what will be provided in the next section.

\section{Complex extension of scaling ansatz}

It would be useful to first recall how the complex extension of $\mu \tau$ interchange symmetry was originally made [27-29]. The $\mu \tau$ interchange invariant $M_{\nu}^{\mu \tau}$ obeys the condition

$\left(G_{3}^{\mu \tau}\right)^{T} M_{v}^{\mu \tau} G_{3}^{\mu \tau}=M_{v}^{\mu \tau}$

with $G_{3}^{\mu \tau}$ given by (2.9). Equation (4.1) forces $M_{v}^{\mu \tau}$ to have the form

$M_{v}^{\mu \tau}=\left(\begin{array}{lll}A & B & B \\ B & C & D \\ B & D & C\end{array}\right)$

with $A, B, C, D$ as mass dimensional complex quantities. It is well known that (4.2) leads to $\theta_{13}=0$ and cannot be accepted as it stands.
This was justified [27-29] by means of a non-standard CPtransformation [34-40] on the $v_{e}$ field which is generally represented as ${ }^{1}$

$v_{L \alpha} \rightarrow i G_{\alpha \beta} \gamma^{0} v_{L \beta}^{C}$

\footnotetext{
${ }^{1}$ It is a theoretically interesting question whether such an extended CPinvariance can arise from an automorphism of a larger flavor symmetry like in the top-down approach of Ref. [42]. But we do not explore this possibility here.
} 
with $G_{\alpha \beta}$ as the matrix element of the flavor symmetry. Equation (4.4) along with (1.1) leads to (4.3) if $G_{\alpha \beta}$ is considered as $G_{3}^{\mu \tau}$. Suffice it to say that (4.3) leads to a complexextended $\mu \tau(C E \mu \tau)$ symmetric form of $M_{\nu}$ : $\tilde{d}=\operatorname{diag}\left(\tilde{d}_{1}, \tilde{d}_{2}, \tilde{d}_{3}\right)$,

where each $\tilde{d}_{i}(i=1,2,3)$ can be +1 or -1 . With $G_{3}=$ $G_{3}^{\text {scaling }},(4.7)$ can be written explicitly:

$$
\begin{gathered}
\left(\begin{array}{ccc}
-\left(U_{e 1}^{\mathrm{CES}}\right)^{*} & -\left(U_{e 2}^{\mathrm{CES}}\right)^{*} & -\left(U_{e 3}^{\mathrm{CES}}\right)^{*} \\
\frac{1-k^{2}}{1+k^{2}}\left(U_{\mu 1}^{\mathrm{CES}}\right)^{*}+\frac{2 k}{1+k^{2}}\left(U_{\tau 1}^{\mathrm{CES}}\right)^{*} & \frac{1-k^{2}}{1+k^{2}}\left(U_{\mu 2}^{\mathrm{CES}}\right)^{*}+\frac{2 k}{1+k^{2}}\left(U_{\tau 2}^{\mathrm{CES}}\right)^{*} \frac{1-k^{2}}{1+k^{2}}\left(U_{\mu 3}^{\mathrm{CES}}\right)^{*}+\frac{2 k}{1+k^{2}}\left(U_{\tau 3}^{\mathrm{CES}}\right)^{*} \\
\frac{2 k}{1+k^{2}}\left(U_{\mu 1}^{\mathrm{CES}}\right)^{*}-\frac{1-k^{2}}{1+k^{2}}\left(U_{\tau 1}^{\mathrm{CES}}\right)^{*} \frac{2 k}{1+k^{2}}\left(U_{\mu 2}^{\mathrm{CES}}\right)^{*}-\frac{1-k^{2}}{1+k^{2}}\left(U_{\tau 2}^{\mathrm{CES}}\right)^{*} \frac{2 k}{1+k^{2}}\left(U_{\mu 3}^{\mathrm{CES}}\right)^{*}-\frac{1-k^{2}}{1+k^{2}}\left(U_{\tau 3}^{\mathrm{CES}}\right)^{*}
\end{array}\right) \\
=\left(\begin{array}{ccc}
\tilde{d}_{1} U_{e 1}^{\mathrm{CES}} & \tilde{d}_{2} U_{e 2}^{\mathrm{CES}} & \tilde{d}_{3} U_{e 3}^{\mathrm{CES}} \\
\tilde{d}_{1} U_{\mu 1}^{\mathrm{CES}} & \tilde{d}_{2} U_{\mu 2}^{\mathrm{CES}} & \tilde{d}_{3} U_{\mu 3}^{\mathrm{CES}} \\
\tilde{d}_{1} U_{\tau 1}^{\mathrm{CES}} & \tilde{d}_{2} U_{\tau 2}^{\mathrm{CES}} & \tilde{d}_{3} U_{\tau 3}^{\mathrm{CES}}
\end{array}\right) .
\end{gathered}
$$

$M_{\nu}^{C E \mu \tau}=\left(\begin{array}{ccc}a & B & B^{*} \\ B & C & d \\ B^{*} & d & C^{*}\end{array}\right)$,

where $a, d$ are real and $B, C$ are complex mass dimensional quantities in general. Once again, since the determinant does not vanish, we take all neutrino masses to be nonzero. The observable consequences of (4.5) are: $\theta_{23}=\pi / 4, \cos \delta=0$, $\alpha, \beta=0$ or $\pi$ while $\theta_{13}$ is in general nonzero. A further extension of this approach has recently been made $[26,41]$ allowing nonmaximal values for $\theta_{23}$ and Dirac CP-violation.

We have derived (3.3), i.e. $\tan \theta_{23}=k^{-1}$, so that atmospheric neutrino mixing is not forced to be strictly maximal. On the other hand, the observed fact that $\tan \theta_{23}$ is not far from unity implies that so is $k$. Our proposed relation, in place of (4.3), is

$$
\left(G_{3}^{\text {scaling }}\right)^{T} M_{\nu} G_{3}^{\text {scaling }}=M_{v}^{*},
$$

with $G_{3}^{\text {scaling }}$ as given in (3.4) and, as stated earlier, in the basis where the charged lepton mass matrix is diagonal and positive. The general form of $M_{v}^{\mathrm{CES}}$, as given in (1.6), follows in consequence. It is important to note that $M_{v}^{\mathrm{CES}}$ of (1.6) has a structure that is quite different from that of either $M_{v}^{\text {SRS }}$ of (1.5) or $M_{v}^{\mathrm{GRS}}$ of (3.8). If all imaginary parts in $M_{v}^{\mathrm{CES}}$ are set equal to zero, a form similar to that of $M_{v}^{\mathrm{GRS}}$ is recovered but with all real entries, while those in $M_{v}^{\mathrm{GRS}}$ of (3.8) are in general complex. Therefore, no special choice in $M_{v}^{\text {CES }}$ can yield $M_{v}^{\mathrm{SRS}}$ or $M_{v}^{\mathrm{GRS}}$ in their respective generalities.

Grimus and Lavoura [27-29] had proved a corollary of complex-extended invariance. This can be stated with respect to a relation such as (4.3) or (4.6) as

$G_{3}^{\text {scaling }} U^{*}=U \tilde{d}$

with $\tilde{d}$ as a diagonal matrix. Once again, $\tilde{d}_{l m}= \pm \delta_{l m}$ if the neutrino masses $m_{1}, m_{2}, m_{3}$ are all nonzero and nondegenerate. The key difference between (4.7) and (2.2) is the complex conjugation of $U$ in the LHS. Let us take
It is evident from (4.9) that the choice $\tilde{d}_{1}=1$ leads to an imaginary $U_{e 1}$ in contradiction with the real $(1,1)$ element of (1.3); this choice is hence excluded. Note that the choice of $U_{\text {PMNS }}$ in (1.3) is simply due to the choice of the weak basis where the neutrino fields are phase rotated. However, in the appendix, we demonstrate that the physical results derived here are basis independent, i.e., the inclusion of an unphysical phase matrix does not impair our predictions. There are now four permitted cases $a, b, c, d$ with the following four combinations allowed for $\tilde{d}$ :

$\tilde{d}_{a} \equiv \operatorname{diag}(-1,1,1)$

$\tilde{d}_{b} \equiv \operatorname{diag}(-1,1,-1)$,

$\tilde{d}_{c} \equiv \operatorname{diag}(-1,-1,1)$,

$\tilde{d}_{d} \equiv \operatorname{diag}(-1,-1,-1)$.

The above can be written compactly as

$$
\begin{aligned}
\tilde{d}_{a, b, c, d} & =\operatorname{diag}(-1, \eta, \xi) \\
\eta_{a, b} & =1, \eta_{c, d}=-1 \\
\xi_{a, c} & =1, \xi_{b, d}=-1 .
\end{aligned}
$$

Comparing with (1.3), we obtain

$$
\begin{aligned}
e^{-i \alpha} & =-\eta, \\
e^{i(2 \delta-\beta)} & =-\xi
\end{aligned}
$$

Thus we are led to the result that $\alpha=\pi, 0$ for $\eta=+1,-1$ respectively; in a similar manner $2 \delta-\beta=\pi, 0$ for $\xi=$ $+1,-1$, respectively. We can derive from (4.9) altogether six independent constraint conditions as linear relations among various elements of $U^{\mathrm{CES}}$ and $\left(U^{\mathrm{CES}}\right)^{*}$. These are listed in Table 1.

More information is obtained by use of the explicit expressions of $U_{l \alpha}^{\mathrm{CES}}$ from (1.3).

Consider the real and imaginary parts of the constraint condition on $U_{\tau 3}^{\text {CES }}$ given in the bottom line in Table 1. Since $c_{13}$ is known to be nonzero, it can be canceled from both 
Table 1 Constraint equations on elements of the mixing matrix

\begin{tabular}{ll}
\hline Element of $U^{\mathrm{CES}}$ & Constraint condition \\
\hline$\mu 1$ & $2 k U_{\mu 1}^{\mathrm{CES}}=\left(1-k^{2}\right) U_{\tau 1}^{\mathrm{CES}}-\left(1+k^{2}\right)\left(U_{\tau 1}^{\mathrm{CES}}\right)^{*}$ \\
$\tau 1$ & $2 k U_{\tau 1}^{\mathrm{CES}}=-\left(1-k^{2}\right) U_{\mu 1}^{\mathrm{CES}}-\left(1+k^{2}\right)\left(U_{\mu 1}^{\mathrm{CES}}\right)^{*}$ \\
$\mu 2$ & $2 k U_{\mu 2}^{\mathrm{CES}}=\left(1-k^{2}\right) U_{\tau 2}^{\mathrm{CES}}+\eta\left(1+k^{2}\right)\left(U_{\tau 2}^{\mathrm{CES}}\right)^{*}$ \\
$\tau 2$ & $2 k U_{\tau 2}^{\mathrm{CES}}=-\left(1-k^{2}\right) U_{\mu 2}^{\mathrm{CES}}+\eta\left(1+k^{2}\right)\left(U_{\mu 2}^{\mathrm{CES}}\right)^{*}$ \\
$\mu 3$ & $2 k U_{\mu 3}^{\mathrm{CES}}=\left(1-k^{2}\right) U_{\tau 3}^{\mathrm{CES}}+\xi\left(1+k^{2}\right)\left(U_{\tau 3}^{\mathrm{CES}}\right)^{*}$ \\
$\tau 3$ & $2 k U_{\tau 3}^{\mathrm{CES}}=-\left(1-k^{2}\right) U_{\mu 3}^{\mathrm{CES}}+\eta\left(1+k^{2}\right)\left(U_{\mu 3}^{\mathrm{CES}}\right)^{*}$ \\
\hline
\end{tabular}

sides. Now, from the respective real and imaginary parts, we have the relations

$$
\begin{aligned}
2 k c_{23} \cos \frac{\beta}{2} & =\left[k^{2}(1+\xi)-1+\xi\right] s_{23} \cos \frac{\beta}{2}, \\
2 k c_{23} \sin \frac{\beta}{2} & =\left[k^{2}(1-\xi)-1-\xi\right] s_{23} \sin \frac{\beta}{2} .
\end{aligned}
$$

Since $\xi^{2}=1$, the product of the above two equations leads to the result

$\sin \beta=0$,

or $\beta=0$ or $\pi$. There are now four options:

$$
\begin{aligned}
& \beta=0, \xi=1 \Rightarrow \tan \theta_{23}=k^{-1}, \\
& \beta=0, \xi=-1 \Rightarrow \tan \theta_{23}=-k, \\
& \beta=\pi, \xi=1 \Rightarrow \tan \theta_{23}=-k, \\
& \beta=\pi, \xi=-1 \Rightarrow \tan \theta_{23}=k^{-1} .
\end{aligned}
$$

The option $\beta=0, \xi=1$ for cases $a$ and $c$, cf. (4.10) and (4.12), as well as $\beta=\pi, \xi=1$ for cases $b$ and $d$, cf. (4.11) and (4.13), yield the scaling relation (3.3) while the other two options require $\tan \theta_{23}$ to equal $-k$. As will be shown below, the latter possibility is inconsistent with other constraint conditions. Our final result on the Majorana phases is that both $\alpha$ and $\beta$ are restricted to be 0 or $\pi$. A combination of information from $0 \nu \beta \beta$ decay, the cosmological upper bound on $\Sigma_{i} m_{i}$ and the effective mass $\Sigma_{i}\left|U_{e i}\right|^{2} m_{i}$ measured in single $\beta$-decay is expected to experimentally constrain [43] these phases.

To proceed, consider the constraint condition on $U_{\tau 2}^{\mathrm{CES}}$ given in the fourth line from the top of Table 1. The corresponding real and imaginary parts, respectively, yield

$$
\begin{aligned}
& 2 k\left[c_{12} s_{23} \cos \frac{\alpha}{2}+s_{12} s_{13} c_{23} \cos \left(\delta+\frac{\alpha}{2}\right)\right] \\
& \quad=\left[1-k^{2}-\eta\left(1+k^{2}\right)\right]\left[c_{12} c_{23} \cos \frac{\alpha}{2}-s_{12} s_{13} s_{23} \cos \left(\delta+\frac{\alpha}{2}\right)\right], \\
& 2 k\left[c_{12} s_{23} \sin \frac{\alpha}{2}+s_{12} s_{13} c_{23} \sin \left(\delta+\frac{\alpha}{2}\right)\right] \\
& \quad=\left[1-k^{2}-\eta\left(1+k^{2}\right)\right]\left[c_{12} c_{23} \sin \frac{\alpha}{2}-s_{12} s_{13} s_{23} \sin \left(\delta+\frac{\alpha}{2}\right)\right] .
\end{aligned}
$$

Let us now take the two cases at hand.
Case 1: $\eta=1, \alpha=\pi$

On utilizing that each of $s_{12}, s_{13}$ and $c_{23}$ is nonzero, one obtains from (4.26) and (4.27) the respective relations

$$
\begin{aligned}
& \left(\tan \theta_{23}-k^{-1}\right) \sin \delta=0, \\
& c_{12}\left(\tan \theta_{23}-k^{-1}\right)+s_{12} s_{13}\left(1+k^{-1} \tan \theta_{23}\right) \cos \delta=0 .
\end{aligned}
$$

Case 2: $\eta=-1, \alpha=0$

It is easy to see that here one obtains the same pair of equations, namely (4.28) and (4.29), but in a reverse sequence.

Equation (4.29) has important implications. If $\tan \theta_{23}$ is put equal to $-k$, instead of $k^{-1}$, one is led to $c_{12}=0$ in contradiction with experiment [9]. Therefore, the two options $\beta=0, \xi=1$ and $\beta=\pi, \xi=-1$ need to be retained with the other two options $\beta=0, \xi=-1$ and $\beta=\pi, \xi=1$ discarded. Now that $\tan \theta_{23}$ does equal $k^{-1}$, i.e. (3.3) holds, from (4.29) we have

$\cos \delta=0$,

i.e. leptonic Dirac CP-violation is maximal with $\delta$ being either $\pi / 2$ or $3 \pi / 2$. However, we are unable to distinguish between these two options since we have no statement on the $\operatorname{sign}$ of $\sin \delta$.

We have checked that (4.30) consistently follows from the remaining four constraint equations of Table 1 and that no new condition emerges. Finally, we are left with four options, as shown in Table 2. Each of these implies (4.30), i.e. the maximality of leptonic Dirac CP-violation which enters via $U_{\text {PMNS. }}$

Table 2 Predictions of the CP phases

\begin{tabular}{llll}
\hline$\tilde{d}$ & $\alpha$ & $\beta$ & $\cos \delta$ \\
\hline$\tilde{d}_{a}=\operatorname{diag}(-1,+1,+1)$ & $\pi$ & 0 & 0 \\
$\tilde{d}_{b}=\operatorname{diag}(-1,+1,-1)$ & $\pi$ & $\pi$ & 0 \\
$\tilde{d}_{c}=\operatorname{diag}(-1,-1,+1)$ & 0 & 0 & 0 \\
$\tilde{d}_{d}=\operatorname{diag}(-1,-1,-1)$ & 0 & $\pi$ & 0 \\
\hline
\end{tabular}


Table 3 Parameters of $M_{v}^{\text {CES }}$ in terms of the parameters of $m_{D}$ and $M_{R}$

$x=-\left(\frac{a^{2}}{M_{1}}+\frac{e^{2}}{M_{2}}+\frac{f^{2}}{M_{3}}\right)$

$y_{1}=\frac{1}{k}\left(\frac{a b_{1}}{M_{1}}+\frac{e c_{1}}{M_{2}}+\frac{f d_{1}}{M_{3}}\right)$

$y_{2}=k\left(\frac{a b_{2}}{M_{1}}+\frac{e c_{2}}{M_{2}}+\frac{f d_{2}}{M_{3}}\right)$

$z_{1}=-\frac{1}{k^{2}}\left(\frac{b_{1}^{2}}{M_{1}}+\frac{c_{1}^{2}}{M_{2}}+\frac{d_{1}^{2}}{M_{3}}\right)+k^{2}\left(\frac{b_{2}^{2}}{M_{1}}+\frac{c_{2}^{2}}{M_{2}}+\frac{d_{2}^{2}}{M_{3}}\right)$

$z_{2}=\frac{2 b_{1} b_{2}}{M_{1}}+\frac{2 c_{1} c_{2}}{M_{2}}+\frac{2 d_{1} d_{2}}{M_{3}}$

$w=\frac{1}{k}\left(\frac{b_{1}^{2}}{M_{1}}+\frac{c_{1}^{2}}{M_{2}}+\frac{d_{1}^{2}}{M_{3}}\right)+k\left(\frac{b_{2}^{2}}{M_{1}}+\frac{c_{2}^{2}}{M_{2}}+\frac{d_{2}^{2}}{M_{3}}\right)$

\section{Origin of neutrino masses from type-I seesaw}

In this section we discuss the realization of the complexextended scaling neutrino mass matrix $M_{v}^{C E S}$ through the type-I seesaw mechanism via three heavy right-handed neutrino fields $N_{l R}(l=1,2,3)$ with a Majorana mass matrix $M_{R}$. We choose a weak basis in which the charged lepton and the right-handed neutrino mass matrices are diagonal and nondegenarate. With $m_{D}$ as the Dirac mass matrix and $M_{R}=\operatorname{diag}\left(M_{1}, M_{2}, M_{3}\right)$, the neutrino mass terms read

$$
-\mathcal{L}_{\text {mass }}^{v, N}=\bar{N}_{l R}\left(m_{D}\right)_{l \alpha} L_{\alpha}+\frac{1}{2} \bar{N}_{l R}\left(M_{R}\right)_{l} \delta_{l m} N_{m R}^{C}+\text { h.c. }
$$

The effective light neutrino mass matrix is given by the standard seesaw relation,

$M_{v}=-m_{D}^{T} M_{R}^{-1} m_{D}$

We represent the $\mathrm{G}$, introduced earlier for left-handed fields, generically by $G_{L}$ and define a corresponding $G_{R}$ for $N_{R}$. The residual CP-transformations on the neutrino fields are defined by [41]

$v_{L \alpha} \rightarrow i\left(G_{L}\right)_{\alpha \beta} \gamma^{0} v_{L \beta}^{C}, N_{R \alpha} \rightarrow i\left(G_{R}\right)_{\alpha \beta} \gamma^{0} N_{R \beta}^{C}$.

The invariance of the mass terms of (5.1) under the CPtransformations defined in (5.3) leads to the relations

$G_{R}^{\dagger} m_{D} G_{L}=m_{D}^{*}, G_{R}^{\dagger} M_{R} G_{R}^{*}=M_{R}^{*}$.

Equations (5.2) and (5.4) together imply $G_{L}^{T} M_{\nu} G_{L}=M_{v}^{*}$. Now, specifying $G_{L}$ by $G_{3}^{\text {scaling }}$, we obtain the key equation

$$
\left(G_{3}^{\text {scaling }}\right)^{T} M_{\nu} G_{3}^{\text {scaling }}=M_{\nu}^{*} \text {. }
$$

Since we choose the right-handed neutrino mass matrix $M_{R}$ to be diagonal, the symmetry matrix $G_{R}$ is diagonal with entries \pm 1 , i.e.

$G_{R}=\operatorname{diag}( \pm 1, \pm 1, \pm 1)$.

Hence there are eight different structures of $G_{R}$. Correspondingly, from the first relation of (5.4), there are eight different structures of $m_{D}$. Unlike the complex transformations of $m_{D}$ and $M_{R}$ in (5.4), we now have real symmetry transformations $G_{R}^{\dagger} m_{D} G_{L}=m_{D}$ and $G_{R}^{\dagger} M_{R} G_{R}^{*}=M_{R}$. It can be shown by tedious algebra that, except for $G_{R}=\operatorname{diag}(-1,-1,-1)$, all other structures of $G_{R}$ are incompatible with scaling symmetry, i.e. cannot generate $M_{v}^{\mathrm{GRS}}$. Thus we take $G_{R}=$ $\operatorname{diag}(-1,-1,-1)$ as the only viable residual symmetry on the right-handed neutrino field. Now, $G_{R}^{\dagger} m_{D} G_{L}=m_{D}^{*}$ can be written as

$m_{D} G_{L}=-m_{D}^{*}$

which is basically a complex extension of the JoshipuraRodejohann result $m_{D} G_{L}=-m_{D}$ [15-21]. In our context, (5.7) can be rewritten as

$m_{D} G_{3}^{\text {scaling }}=-m_{D}^{*}$.

The most general $m_{D}$ that satisfies (5.8) is

$m_{D}^{\mathrm{CES}}=\left(\begin{array}{ccc}a & b_{1}+i b_{2}-b_{1} / k+i b_{2} k \\ e & c_{1}+i c_{2}-c_{1} / k+i c_{2} k \\ f & d_{1}+i d_{2}-d_{1} / k+i d_{2} k\end{array}\right)$,

where $a, b_{1,2}, c_{1,2}, d_{1,2}, e$, and $f$ are arbitrary real mass dimensional quantities. Using (5.2), $M_{v}^{\mathrm{CES}}$ of (1.6) is obtained with the parameters as given in Table 3 . Some detailed interesting consequences of $m_{D}^{\mathrm{CES}}$, specifically with respect to leptogenesis, will be studied elsewhere.

We notice that, for both types of ordering, the neutrino masses become hierarchical, i.e. $m_{2,1} \ll m_{3}$ for normal ordering and $m_{2,1} \gg m_{3}$ for inverted ordering, for low values of the lightest neutrino mass. However, they tend toward quasi-degeneracy $m_{1} \sim m_{2} \sim m_{3}$ as the latter increases to its permitted maximum value $\sim 0.07 \mathrm{eV}$. This is clear from the mass bands shown in Fig. 1.

\section{Phenomenological constraints and consequences}

We need to numerically pin down the mass dimensional six real parameters $x, y_{1}, y_{2}, z_{1}, z_{2}$ and $w$ of $M_{v}^{\mathrm{CES}}$ by inputting the $3 \sigma$ ranges of quantities measured in neutrino oscillation experiments. To that end, we use the values from a recent global analysis [9]. In addition, we use the cosmological upper limit [44] of $0.23 \mathrm{eV}$ on the sum $m_{1}+m_{2}+m_{3}$ of the masses of the neutrinos.

These input numbers are shown in Table 4 . In terms of output, we obtain the $3 \sigma$ allowed intervals of the above mentioned six real parameters and from those the $3 \sigma$ allowed ranges of the individual neutrino masses $m_{1}, m_{2}, m_{3}$. Both types of neutrino mass ordering, normal as well as inverted, are found to be allowed. All these values are listed in Tables 5 and 6 respectively for the two separate categories of mass ordering. 
Fig. 1 Plots of the mass band for normal $(l e f t)$ and inverted (right) mass ordering. We have choosen to plot the lightest eigenvalue also in the ordinate to bring three mass bands together. Color code: green $\left(m_{3}\right)$, red $\left(m_{2}\right)$ and blue $\left(m_{1}\right)$
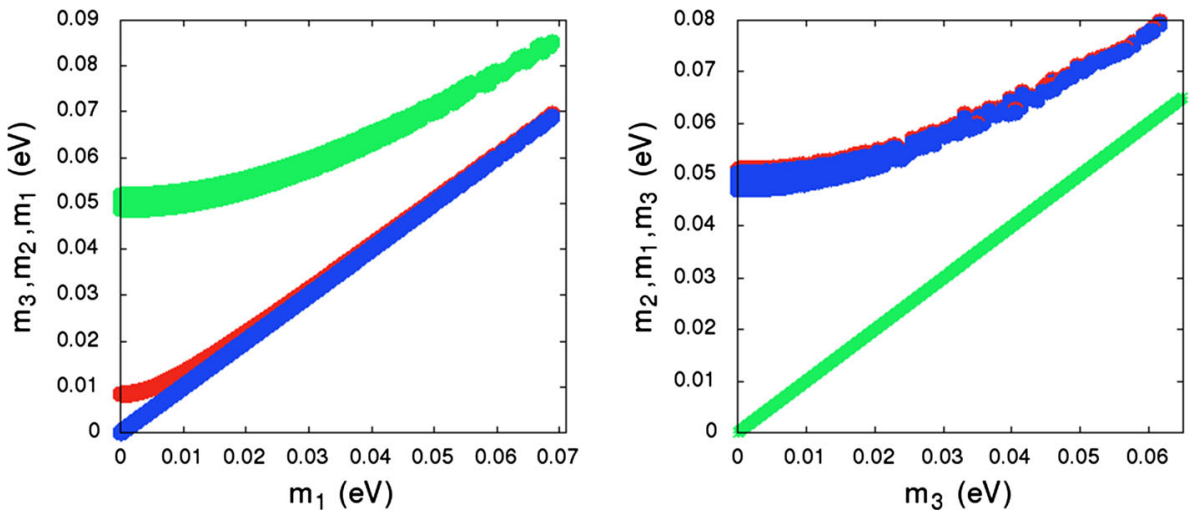

Table 4 Input values used

\begin{tabular}{llllll}
\hline$\theta_{12}$ degrees & $\theta_{23}$ degrees & $\theta_{13}$ degrees & $\Delta m_{21}^{2} 10^{-5} \mathrm{eV}^{2}$ & $\left|\Delta m_{31}^{2}\right| 10^{-3}\left(\mathrm{eV}^{2}\right)$ & $\Sigma_{i} m_{i}(\mathrm{eV})$ \\
\hline $31.29-35.91$ & $38.3-53.3$ & $7.87-9.11$ & $7.02-8.09$ & $2.32-2.59$ & $<0.23$ \\
\hline
\end{tabular}

Table 5 Output values obtained for normal mass ordering with the best fit $m$ 's given within brackets

\begin{tabular}{|c|c|c|c|c|c|}
\hline$x(\mathrm{eV})$ & $y_{1}(\mathrm{eV})$ & $y_{2}(\mathrm{eV})$ & $z_{1}(\mathrm{eV})$ & $z_{2}(\mathrm{eV})$ & $w(\mathrm{eV})$ \\
\hline$-0.20-+0.21$ & $-0.12-+0.11$ & $-0.05-+0.05$ & $-0.17-+0.17$ & $-0.18-+0.17$ & $-0.16-+0.15$ \\
\hline \multicolumn{2}{|l|}{$m_{1}(\mathrm{eV})$} & \multicolumn{2}{|l|}{$m_{2}(\mathrm{eV})$} & \multicolumn{2}{|l|}{$m_{3}(\mathrm{eV})$} \\
\hline \multicolumn{2}{|c|}{$9.2 \times 10^{-5}-0.071(0.052)$} & \multicolumn{2}{|c|}{$0.01-0.077(0.054)$} & \multicolumn{2}{|c|}{$0.051-0.082(0.072)$} \\
\hline
\end{tabular}

Table 6 Output values obtained for inverted mass ordering with the best fit $m$ 's given within brackets

\begin{tabular}{|c|c|c|c|c|c|}
\hline$x(\mathrm{eV})$ & $y_{1}(\mathrm{eV})$ & $y_{2}(\mathrm{eV})$ & $z_{1}(\mathrm{eV})$ & $z_{2}(\mathrm{eV})$ & $w(\mathrm{eV})$ \\
\hline$-0.44-+0.46$ & $-0.16-+0.16$ & $-0.14-+0.14$ & $-0.01-+0.01$ & $-0.01-+0.01$ & $-0.05-+0.06$ \\
\hline$m_{1}(\mathrm{eV})$ & & $m_{2}(\mathrm{eV})$ & & $m_{3}(\mathrm{eV})$ & \\
\hline $0.049-0.079(0.068)$ & & $0.051-0.085(0.069)$ & & $8.2 \times 10^{-5}-0$ & 48) \\
\hline
\end{tabular}

Neutrinoless double beta decay $0 \nu \beta \beta$

This is the lepton number violating process

$(A, Z) \longrightarrow(A, Z+2)+2 e^{-}$

with no final state neutrinos. An observation of the decay will confirm the Majorana nature of neutrinos which is yet to be established. The corresponding half-life $[45,46]$ is given by

$\frac{1}{T_{1}^{0 v} / 2}=G_{0 v}\left|M_{0 v}\right|^{2}\left|M_{e e}^{v}\right|^{2} m_{e}^{-2}$

where $G_{0 v}$ is a phase space factor, $M_{0 v}$ is the nuclear matrix element (NME), $m_{e}$ is the electron mass and finally $\left|M_{e e}^{v}\right|$ is the $(1,1)$ element of $M^{v}$ which can also be written as $\Sigma_{i} m_{i} U_{e i}^{2}$. Following the PDG parametrization of the mixing matrix $U_{\mathrm{PMNS}}$, one can write $M_{e e}^{v}$ as

$M_{e e}^{\nu}=c_{12}^{2} c_{13}^{2} m_{1}+s_{12}^{2} c_{13}^{2} m_{2} e^{i \alpha}+s_{13}^{2} m_{3} e^{i(\beta-2 \delta)}$.

There are several ongoing experiments which have put significant upper limits on $\left|M_{e e}^{v}\right|$. Some recent experiments like KamLAND-Zen [47] and EXO [48] have improved this upper bound to $0.35 \mathrm{eV}$. However, the most significant upper bound on $\left|M_{e e}^{v}\right|$ to date is put by GERDA phase-I data [49] to be $0.22 \mathrm{eV}$; this is likely to be lowered by GERDA phase -II data [50] to around $0.098 \mathrm{eV}$.

In our model there are four sets of values of the $\mathrm{CP}$ violating phases $\alpha$ and $\beta$ for each neutrino mass ordering. Since $\left|M_{e e}^{v}\right|$ is sensitive to the CP phases, we get four different plots for each mass ordering as shown in Fig. 2. The same plots are valid for both types of mass ordering provided the horizontal axis is taken to represent the lightest neutrino mass $m_{1}$ or $m_{3}$-depending on the ordering.

As mentioned earlier, we have used the upper bound of $0.23 \mathrm{eV}$ on $\Sigma_{i} m_{i}$. These plots lead to upper bounds on the lightest neutrino mass for both cases of mass ordering. For hierarchical neutrinos, $\left|M_{e e}^{v}\right|$ is found to lead to an upper limit which is below the reach of the GERDA phase-II data. The latter appears close to being obtainable only for a quasidegenerate neutrino mass spectrum ( $\left.m_{\text {lightest }}>0.07 \mathrm{eV}\right)$. However, the value predicted in our model could be probed by a 

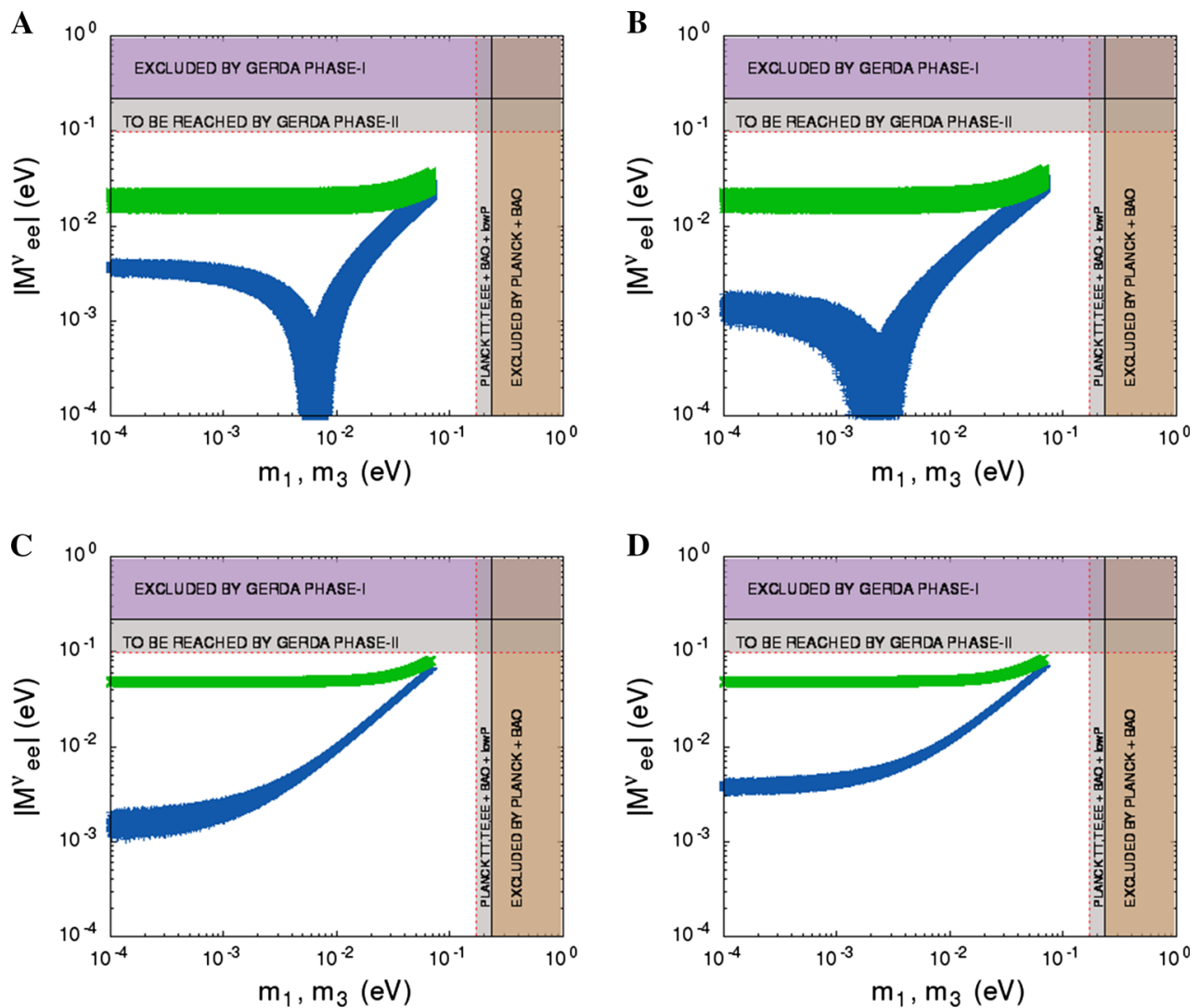

Fig. 2 Plot of $\left|M_{e e}^{v}\right|$ vs. the lightest neutrino mass: the top two figures represent Case A (left) and Case B (right) while the figures in the lower panel represent Case C (left) and Case D (right)

combination of GERDA and MAJORANA experiments [51]. In order to explain the nature of the plots analytically, let us first consider the inverted mass ordering: In this case, with the approximations $m_{3} \simeq 0$ and $m_{1} \simeq m_{2}$, the probed effective mass $\left|M_{e e}^{v}\right|$ simplifies to

$$
\begin{aligned}
\left|M_{e e}^{v}\right|= & \sqrt{\left|\Delta m_{32}\right|^{2}} c_{13}^{2}\left[\left\{1-s_{12}^{2}(1-\cos \alpha)\right\}^{2}\right. \\
& \left.+s_{12}^{4} \sin ^{2} \alpha\right]^{1 / 2} .
\end{aligned}
$$

Clearly, $\left|M_{e e}^{\nu}\right|$ is insensitive to the phases $\beta$ and $\delta$. On the other hand, for $\alpha=0$ and $\pi$ (6.4) simplifies to

$\left|M_{e e}^{v}\right|=\sqrt{\left|\Delta m_{32}\right|^{2}} c_{13}^{2}$

and

$\left|M_{e e}^{v}\right|=\sqrt{\left|\Delta m_{32}\right|^{2}} c_{13}^{2}\left[\left\{1-2 s_{12}^{2}\right\}^{2}\right]$,

respectively. Hence, for $\alpha=\pi$ (cases A, B ), $\left|M_{e e}^{v}\right|$ is suppressed as compared to the case $\alpha=0(\mathrm{C}, \mathrm{D})$. For a normal mass ordering, in addition to the $s_{13}$ suppression, there is a significant interference between the first two terms, thus lowering the value of $\left|M_{e e}^{v}\right|$. However, if $\alpha=0$, the first two terms interfere constructively and then we obtain a lower bound $\left(\sim 10^{-3} \mathrm{eV}\right.$ for Case $\mathrm{C}$ and $\sim 5 \times 10^{-3} \mathrm{eV}$ for Case D) despite this being a case of normal mass ordering. This is one of the remarkable results of the present analysis. On the other hand, for $\alpha=\pi$, the first two terms interfere destructively, for the case of a normal mass ordering; consequently, a sizable cancelation between them brings down the value of $\left|M_{e e}^{v}\right|$ and results in the kinks shown by the lower curves in the top two figures.

\section{CP asymmetry in neutrino oscillations}

Here we discuss the determination of our predicted maximal Dirac CP-violating phase $\delta$ by means of neutrino oscillation studies. This $\delta$ will show up in the asymmetry parameter $A_{\alpha \beta}$, defined as

$A_{\alpha \beta}=P\left(v_{\alpha} \rightarrow v_{\beta}\right)-P\left(\overline{v_{\alpha}} \rightarrow \overline{v_{\beta}}\right)$,

where $\alpha, \beta=(e, \mu, \tau)$ are flavor indices and the $P$ are transition probabilities. Let us consider first $v_{\mu} \rightarrow v_{e}$ oscillation in vacuum. The transition probability can now be written (with the superscript zero indicating oscillations in vacuum) as

$$
\begin{aligned}
P_{\mu e}^{0} \equiv & P^{0}\left(v_{\mu} \rightarrow v_{e}\right)=P_{\mathrm{atm}}^{0}+P_{\mathrm{sol}}^{0} \\
& +2 \sqrt{P_{\mathrm{atm}}^{0} \sqrt{P_{\mathrm{sol}}^{0}} \cos \left(\Delta_{32}+\delta\right),}
\end{aligned}
$$



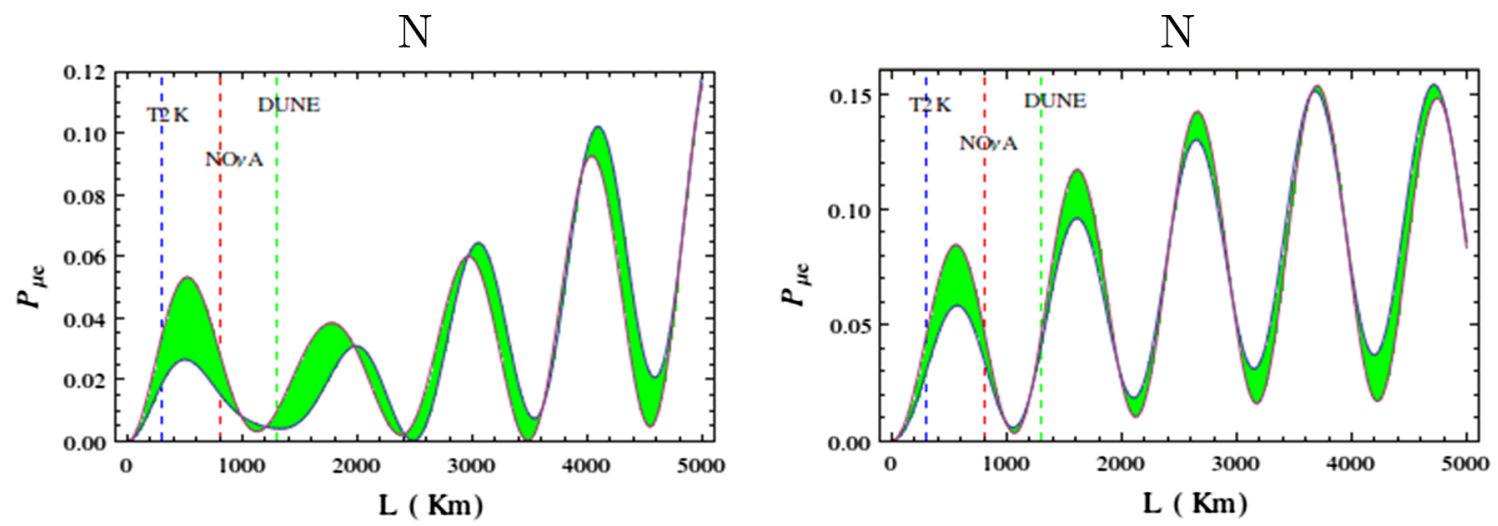

I
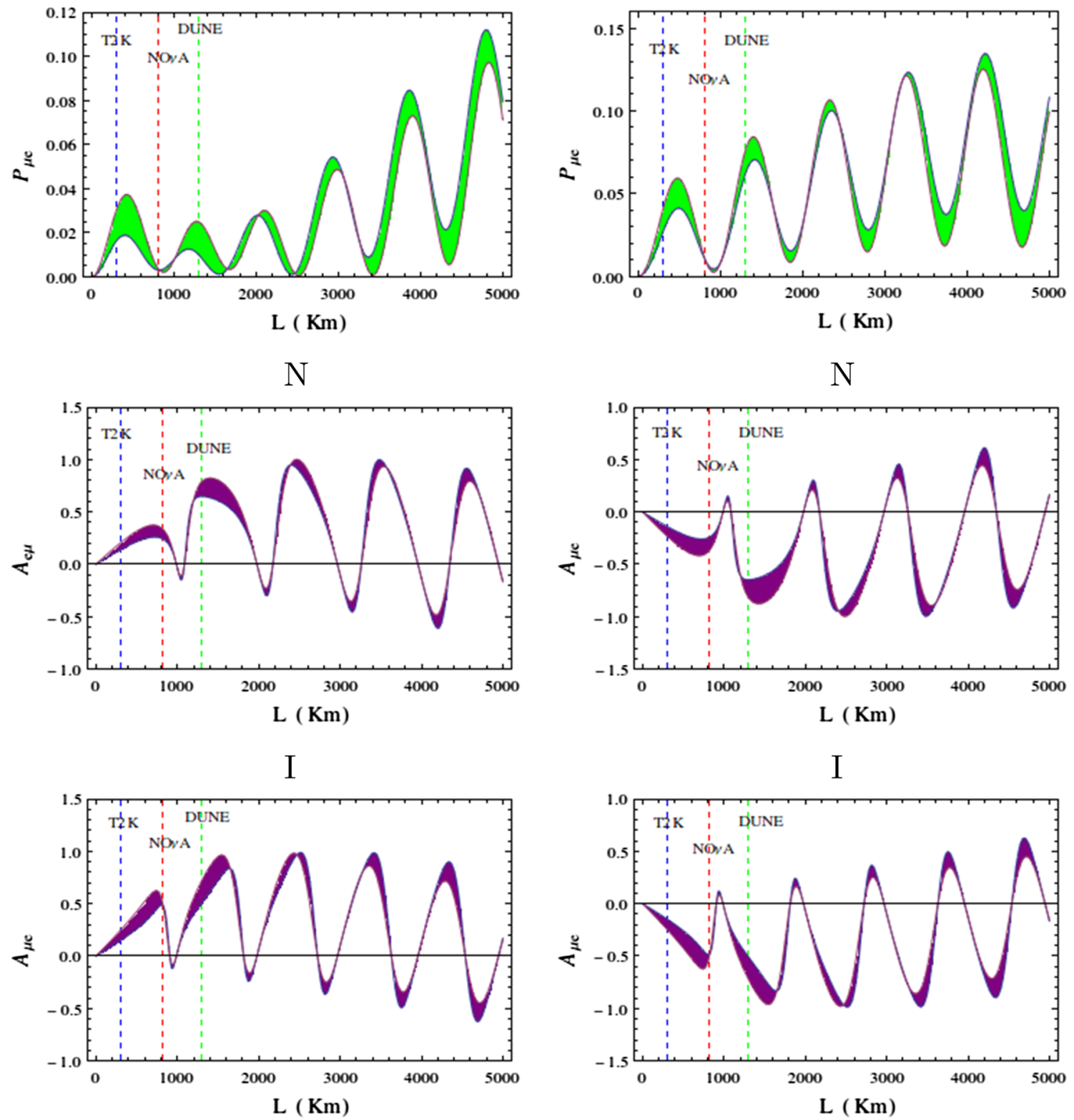

Fig. 3 Plots of the transition probability $\left(P_{\mu e}\right)$ and CP-asymmetry parameter $\left(A_{\mu e}\right)$ with baseline length $L$ for $\delta=\pi / 2$ (left panel) and $\delta=3 \pi / 2$ (right panel) with $E=1 \mathrm{GeV}$. Cases for normal (inverted)

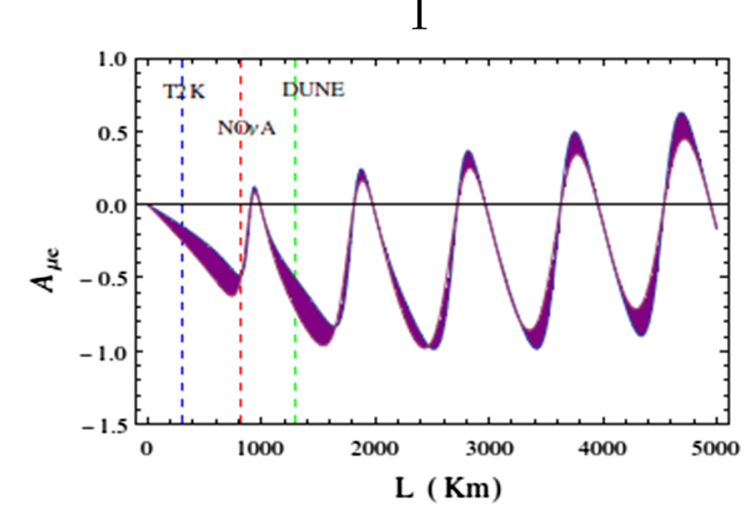

mass ordering have been labeled on top by $N(I)$. The bands are caused by the atmospheric mixing angle $\theta_{23}$ being allowed to vary within the $3 \sigma$ region while the other parameters are kept at their best fit values 

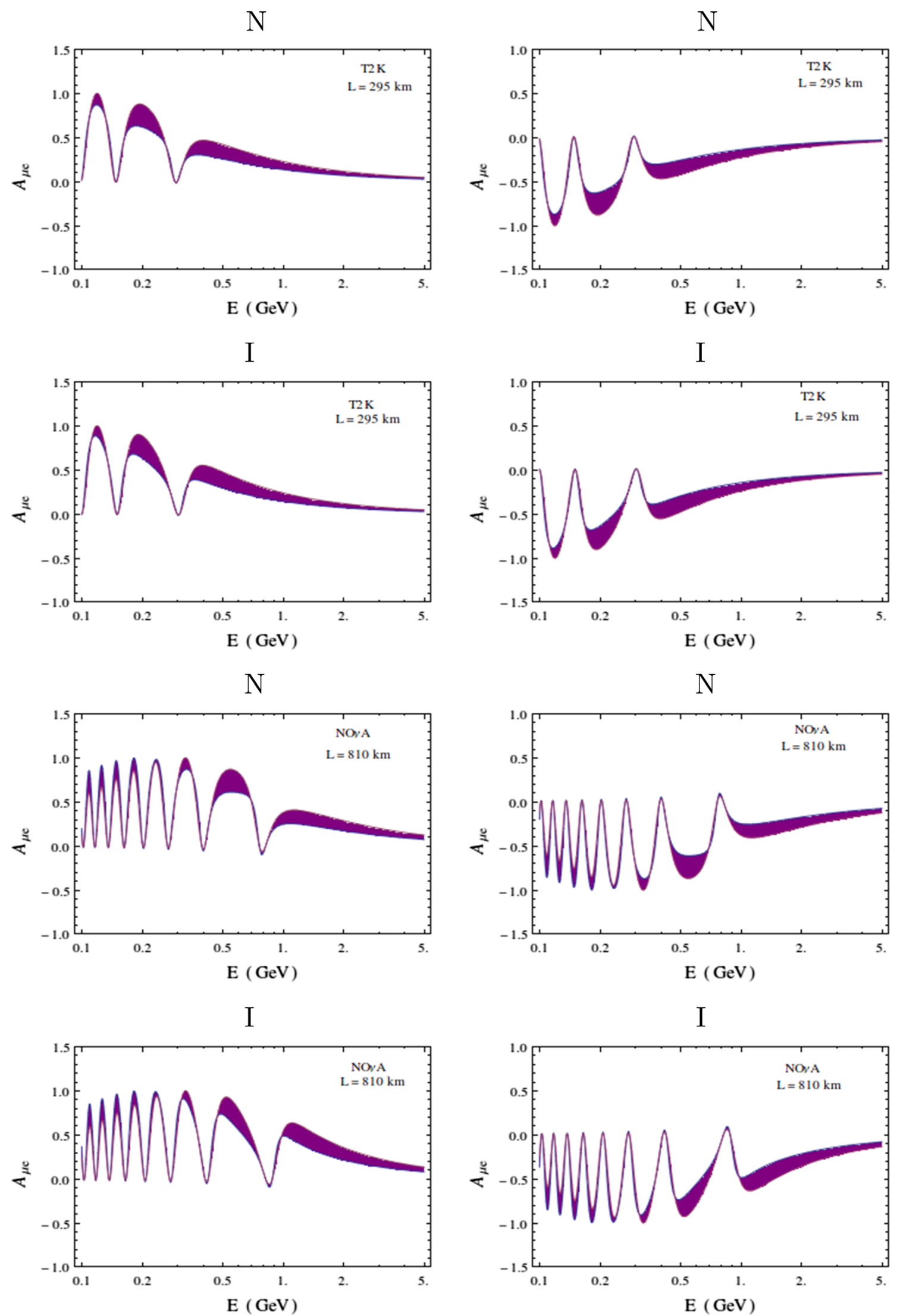

Fig. 4 Plots of the CP-asymmetry parameter $A_{\mu e}$ against beam energy $E$ for $\delta=\pi / 2$ (left panel) and $\delta=3 \pi / 2$ (right panel) for various experiments as shown. Cases for normal (inverted) mass ordering have been

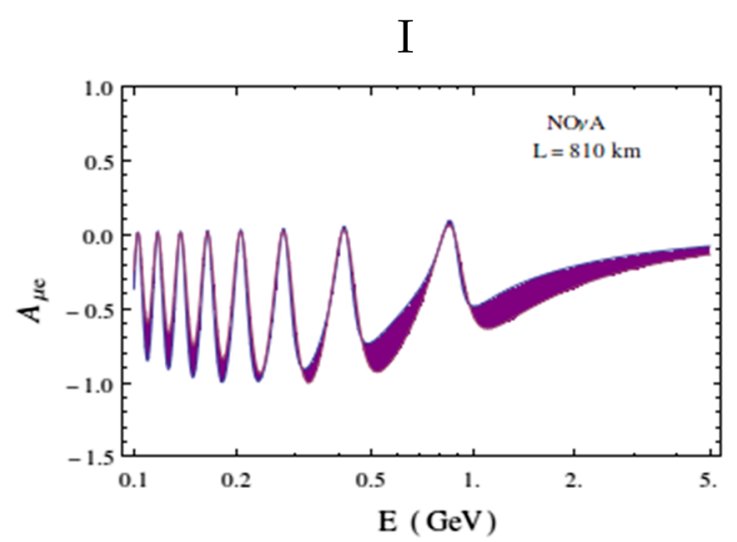

labeled on top by $N(I)$. The atmospheric mixing angle $\theta_{23}$ is allowed to vary within the $3 \sigma$ region, leading to the bands, while the other parameters are kept at their best fit values 

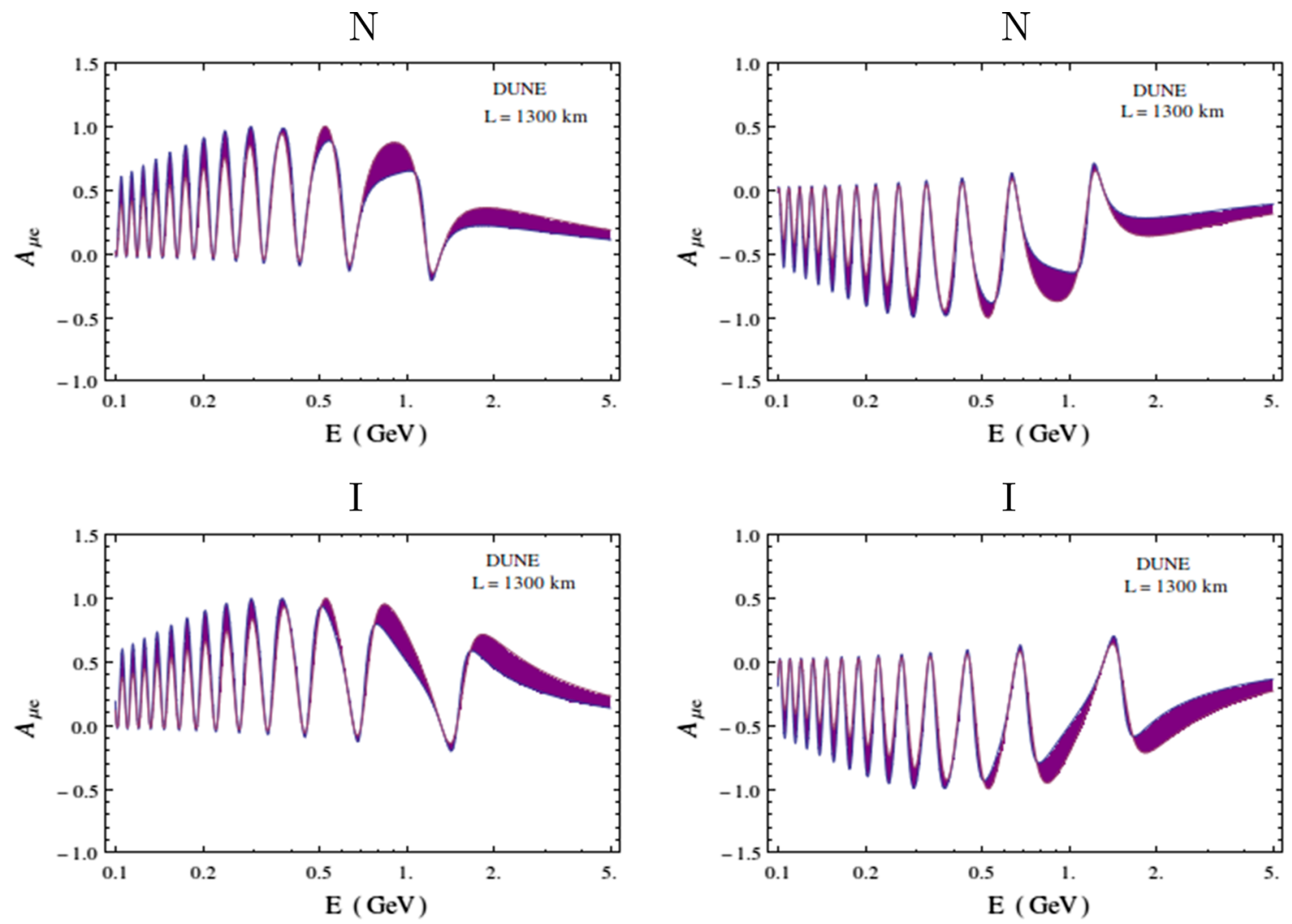

Fig. 4 continued

where $\Delta_{i j}=\frac{\Delta m_{i j}^{2} L}{4 E}$ is the kinematic phase factor ( $L$ being the baseline length and $E$ being the beam energy) and $P_{\text {atm }}^{0}, P_{\text {sol }}^{0}$ are, respectively, defined as

$\sqrt{P_{\mathrm{atm}}^{0}}=\sin \theta_{23} \sin 2 \theta_{13} \sin \Delta_{31}$,

$\sqrt{P_{\mathrm{sol}}^{0}}=\cos \theta_{23} \cos \theta_{13} \sin 2 \theta_{12} \sin \Delta_{21}$.

Table 7 Predictions of the CP phases for $\tilde{d}_{1}=1$

\begin{tabular}{llll}
\hline$\tilde{d}$ & $\alpha$ & $\beta$ & $\cos \delta$ \\
\hline$\tilde{d}_{e}=\operatorname{diag}(-1,+1,+1)$ & 0 & 0 & 0 \\
$\tilde{d}_{f}=\operatorname{diag}(-1,+1,-1)$ & 0 & $\pi$ & 0 \\
$\tilde{d}_{g}=\operatorname{diag}(-1,-1,+1)$ & $\pi$ & 0 & 0 \\
$\tilde{d}_{h}=\operatorname{diag}(-1,-1,-1)$ & $\pi$ & $\pi$ & 0 \\
\hline
\end{tabular}

For an antineutrino beam, $\delta$ is replaced by $-\delta$ and thus we have

With our prediction $\cos \delta=0,(6.12)$ can be rewritten as

$$
\begin{aligned}
\bar{P}_{\mu e}^{0} \equiv & P^{0}\left(\overline{v_{\mu}} \rightarrow \overline{v_{e}}\right)=P_{\mathrm{atm}}^{0}+P_{\mathrm{sol}}^{0} \\
& +2 \sqrt{P_{\mathrm{atm}}^{0}} \sqrt{P_{\mathrm{sol}}^{0}} \cos \left(\Delta_{32}-\delta\right) .
\end{aligned}
$$

Now the CP-asymmetry parameter $A_{\mu e}^{0}$ in vacuum [52] can be calculated as

$$
\begin{aligned}
A_{\mu e}^{0} & =\frac{P_{\mu e}^{0}-\bar{P}_{\mu e}^{0}}{P_{\mu e}^{0}+\bar{P}_{\mu e}^{0}} \\
& =\frac{2 \sqrt{P_{\mathrm{atm}}^{0}} \sqrt{P_{\mathrm{sol}}^{0}} \sin \Delta_{32} \sin \delta}{P_{\mathrm{atm}}^{0}+P_{\mathrm{sol}}^{0}+2 \sqrt{P_{\mathrm{atm}}^{0}} \sqrt{P_{\mathrm{sol}}^{0}} \cos \Delta_{32} \cos \delta} .
\end{aligned}
$$

$A_{\mu e}^{0}= \pm \frac{2 \sqrt{P_{\mathrm{atm}}^{0}} \sqrt{P_{\mathrm{sol}}^{0}} \sin \Delta_{32}}{P_{\mathrm{atm}}^{0}+P_{\mathrm{sol}}^{0}}$,

with a $+(-)$ sign for $\delta=\pi / 2(3 \pi / 2)$.

In order to realistically describe neutrino oscillations in long baseline experiments, matter effects in neutrino propagation through the earth need to be taken into account. In that case $P_{\mathrm{atm}}^{0}$ and $P_{\mathrm{sol}}^{0}$ will be modified to

$$
\begin{aligned}
& \sqrt{P_{\mathrm{atm}}}=\sin \theta_{23} \sin 2 \theta_{13} \frac{\sin \left(\Delta_{31}-a L\right)}{\Delta_{31}-a L} \Delta_{31}, \\
& \sqrt{P_{\mathrm{sol}}}=\cos \theta_{23} \cos \theta_{13} \sin 2 \theta_{12} \frac{\sin a L}{a L} \sin \Delta_{21},
\end{aligned}
$$


respectively. Here $a=G_{F} N_{e} / \sqrt{2}$ with $G_{F}$ as the Fermi constant and $N_{e}$ as the number density of electrons in the medium of propagation. An approximate value of $a$ for the earth is $3500 \mathrm{~km}^{-1}$ [52]. Now the same formulas for $P_{\mu e}$, $\bar{P}_{\mu e}$ and $A_{\mu e}$ will hold as in (6.8), (6.11) and (6.12) but with $P_{\text {atm }}^{0}$ and $P_{\text {sol }}^{0}$ replaced by $P_{\text {atm }}$ and $P_{\text {sol }}$, respectively.

In Fig. 3 we plot $P_{\mu e}$ and $A_{\mu e}$ against the baseline length $\mathrm{L}$ in the two cases $\delta=\pi / 2$ and $\delta=3 \pi / 2$ for both normal and inverted mass ordering. The lengths corresponding to $\mathrm{T} 2 \mathrm{~K}$, NO $\nu \mathrm{A}$ and DUNE are indicated in these figures. In Fig. 4 the $\mathrm{CP}$ asymmetry $A_{\mu e}$ is plotted against the beam energy $\mathrm{E}$ again for the cases $\delta=\pi / 2$ and $\delta=3 \pi / 2$ separately for the three above cited experiments; both normal and inverted mass ordering cases are included. As expected, $A_{\mu e}$ has opposite signs for $\delta=\pi / 2$ and $\delta=3 \pi / 2$. It is further interesting that the extrema of the CP-asymmetry parameter exhibit opposite behavior as a function of $\mathrm{E}$ for $\delta=\pi / 2$ and $\delta=3 \pi / 2$.

\section{Summary}

In this paper we have proposed a complex extension of the scaling ansatz for the neutrino Majorana mass matrix $M^{v}$. To that end, we have made use of the residual $\mathbb{Z}_{2} \times \mathbb{Z}_{2}^{\text {scaling }}$ symmetry of $M^{v}$ by obtaining the representation $G_{3}^{\text {scaling }}$ from the original simple scaling ansatz on $M^{v}$. The resultant form of the neutrino Majorana matrix is given by $M_{v}^{\mathrm{CES}}$ of (1.6). We have shown that it admits nonzero values of all the physical neutrino masses as well as both normal and inverted types of mass ordering. We have shown how a nonvanishing $\theta_{13}$ emerges from $M_{v}^{\text {CES }}$. The additional result $k^{-1}=\tan \theta_{23}, k$ being the real positive scaling factor, has also been derived. Dirac CP-violation has been shown to be maximal with $\cos \delta=0$, while Majorana CP-violation has been demonstrated to be absent with $\alpha, \beta=0$ or $\pi$. The type-I seesaw mechanism which yields nonzero neutrino masses within our scheme has also been constructed. Phenomenological implications for both $0 \nu \beta \beta$ decay and neutrino/antineutrino oscillation studies at long baselines have been worked out and projections made that will be testable in forthcoming experiments.

Acknowledgements We thank P. S. Bhupal Dev and Ketan M. Patel for their comments. RS and AG acknowledge the Department of Atomic Energy, Govt. of India, for financial support. The work of PR has been supported by Indian National Science Academy.

Open Access This article is distributed under the terms of the Creative Commons Attribution 4.0 International License (http://creativecomm ons.org/licenses/by/4.0/), which permits unrestricted use, distribution, and reproduction in any medium, provided you give appropriate credit to the original author(s) and the source, provide a link to the Creative Commons license, and indicate if changes were made.

Funded by SCOAP ${ }^{3}$.
Appendix: Derivation of the results on CP-violation even with the inclusion of the unphysical matrix

As mentioned in Sect. 1, our calculations have been done in a weak basis where the unphysical phases are absorbed in the neutrino fields. However, one can also reproduce our results including this unphysical phase matrix in the calculation. In that case the $U_{\text {PMNS }}$ of (1.3) can be written as

$U_{\mathrm{PMNS}}=P_{\phi} U$,

with $P_{\phi}=$ diag. $\left(e^{i \phi}, 1,1\right)$. Note that there is only a single unphysical phase in the phase matrix $P_{\phi}$, since the symmetry under consideration dictates $M_{v}^{\mathrm{CES}}$ in (1.6) to contain seven real parameters which correspond to three nonzero masses, three mixing angles and an unphysical phase. Now for $\tilde{d}_{1}=$ -1 , Eq. (4.9) and the (1,1) element of $U_{\mathrm{PMNS}}$ in (A.1) give

$e^{-2 i \phi}=1$,

therefore, $\phi=0$ or $\pi$. From the $(1,2)$ element we get

$e^{-i(\alpha+2 \phi)}=-\eta$.

Thus for both values of $\phi$, (A.3) leads to (4.17); therefore, for each $\tilde{d}$ matrix with $\tilde{d}_{1}=-1$, the prediction for $\alpha$, i.e., $\alpha=0$ or $\pi$ remain the same. Now, following the same way as in Sect. 4, the results presented in Table 2 can be reproduced.

Unlike the previous case, now $\tilde{d}_{1}=1$ cannot be ruled out. In this case, from the $(1,1)$ element of $U_{\text {PMNS }}$ in (A.1), we get $\phi=\pi / 2$ or $3 \pi / 2$. Now, for both values of $\phi$, Eq. (A.3) with $\eta=1$ leads to $\alpha=0$, and with $\eta=-1$ leads to $\alpha=\pi$. Since the predictions for $\alpha$ remain the same, so do the other parameters which are solved exactly in the same way as in Sect. 4, by use of both real and imaginary parts of the relevant complex equations. We put the more general statements regarding the CP phases for each $\tilde{d}$ with $\tilde{d}_{1}=1$ in Table 7. In comparison with Table 2, the values of $\alpha$ have changed relative to those of $\beta$, but the final result that both $\alpha$ and $\beta$ are either 0 or $\pi$ remains the same, though the value of $\tilde{d}_{1}$ has changed.

\section{References}

1. S.F. King, J. Phys. G 42, 123001 (2015)

2. S. Verma, Adv. High Energy Phys. 2015, 385968 (2015)

3. M. Fukugita, T. Yanagida, Phys. Lett. B 174, 45 (1986)

4. S. Davidson, E. Nardi, Y. Nir, Phys. Rept. 486, 105 (2008)

5. C. Hagedorn, E. Molinaro. arXiv:1602.04206 [hep-ph]

6. K. Abe et al. [T2K Collaboration], Phys. Rev. D 91(7), 072010 (2015)

7. J. Bian [NOvA Collaboration]. arXiv:1510.05708 [hep-ex]

8. C. Kachulis, "SK Atmospheric Neutrino Results", talk given at the Meeting of the Division of Particles and Fields of the American Physical Society, August 4-8, 2015. https://indico. 
cern.ch/event/361123/session/2/contribution/348/attachments/ 1136004/1625868/SKatmospheric_kachulis_dpf2015

9. M.C. Gonzalez-Garcia, M. Maltoni, T. Schwetz. arXiv:1512.06856 [hep-ph]

10. S. Dell'Oro, S. Marcocci, F. Vissani, Proceedings, 16th International Workshop on Neutrino Telescopes (Neutel 2015). PoS NEUTEL 2015, 069 (2015)

11. G.C. Branco, L. Lavoura, J.P. Silva, CP violation (Clarendon Press, Oxford, 1999)

12. K.A Olive et al. [Particle Data Group], Chin. Phys. C 38, 090001 (2014)

13. L. Lavoura, Phys. Rev. D 62, 093011 (2000)

14. R.N. Mohapatra, W. Rodejohann, Phys. Lett. B 644, 59 (2007)

15. M.S. Berger, S. Santana, Phys. Rev. D 74, 113007 (2006)

16. A. Blum, R.N. Mohapatra, W. Rodejohann, Phys. Rev. D 76, 053003 (2007)

17. A.S. Joshipura, W. Rodejohann, Phys. Lett. B 678, 276 (2009)

18. B. Adhikary, M. Chakraborty, A. Ghosal, Phys. Rev. D 86, 013015 (2012)

19. M. Chakraborty, H.Z. Devi, A. Ghosal, Phys. Lett. B 741, 210 (2015)

20. A. Ghosal, R. Samanta, JHEP 1505, 077 (2015)

21. R. Samanta, M. Chakraborty, A. Ghosal, Nucl. Phys. B 904, 86 (2016)

22. B.Z Hu et al. (Daya Bay Collaboration), Phys. Rev. Lett. 115, 111802 (2015) (references therein)

23. C.S. Lam, Phys. Lett B 656, 193 (2007)

24. C.S. Lam, Phys. Rev. Lett. 101, 121602 (2008)

25. C.S. Lam, Phys. Rev. D 78, 073015 (2008)

26. P. Chen, G.J. Ding, F. Gonzalez-Canales, J.W.F. Valle, Phys. Lett. B 753, 644 (2016)

27. W. Grimus, L. Lavoura, Acta Phys. Polon. B 34, 5393 (2003)

28. W. Grimus, L. Lavoura, Phys. Lett. B 579, 113 (2004)

29. W. Grimus, L. Lavoura, Fortsch. der Phys. 61, 535 (2013)

30. W. Grimus, A.S. Joshipura, S. Kaneko, L. Lavoura, H. Sawanaka, M. Tanimoto, Nucl. Phys. B 713, 151 (2005)

31. S.F Ge, D.A. Dicus, W.W Repko, Phys. Rev. D 83, 093007 (2011)
32. S.F Ge, D.A. Dicus, W.W Repko, Phys. Lett. B 702, 220 (2011)

33. S.F Ge, D.A. Dicus, W.W Repko, Phys. Rev Lett. 108, 041801 (2012)

34. G. Ecker, W. Grimus, H. Neufeld, J. Phys. A 20, L807 (1987)

35. G. Ecker, W. Grimus, H. Neufeld, Int. J. Mod. Phys. A 3, 603 (1988)

36. W. Grimus, M.N. Rebelo, Phys. Rept. 281, 239 (1997)

37. S. Gupta, A.S. Joshipura, K.M. Patel, Phys. Rev. D 85, 031903 (2012)

38. G.J. Ding, S.F. King, A.J. Stuart, JHEP 1312, 006 (2013)

39. C. Hagedorn, A. Meroni, E. Molinaro, Nucl. Phys. B 891, 499 (2015)

40. P. Chen, C.Y. Yao, G.J. Ding, Phys. Rev. D 92(7), 073002 (2015)

41. P. Chen, G.J. Ding, S.F. King, JHEP 1603, 206 (2016)

42. F. Feruglio, C. Hagedorn, R. Ziegler, JHEP 1307, 027 (2013)

43. H. Minakata, H. Nunokawa, A.A. Quiroga, PTEP 2015, 033 B03 (2015)

44. P.A.R. Ade et al. [Planck Collaboration]. arXiv:1502.01589 [astroph.CO]

45. W. Rodejohann, Int. J. Mod. Phys. E 20, 1833 (2011)

46. P.S.B. Dev, S. Goswami, M. Mitra, W. Rodejohann, Phys. Rev. D 88, 091301 (2013)

47. K. Asakura et al. [KamLAND-Zen Collaboration], Nucl. Phys. A 946, 171 (2016)

48. M. Auger et al. [EXO-200 Collaboration], Phys. Rev. Lett. 109, 032505 (2012)

49. M. Agostini et al. [GERDA Collaboration], Phys. Rev. Lett. 111(12), 122503 (2013). doi:10.1103/PhysRevLett.111.122503. arXiv:1307.4720 [nucl-ex]

50. B. Majorovits [GERDA Collaboration], AIP Conf. Proc. 1672, 110003 (2015). arXiv:1506.00415 [hep-ex]

51. N. Abgrall et al. [Majorana Collaboration], Adv. High Energy Phys. 2014, 365432 (2014)

52. H. Nunokawa, S. Parke, J.W. Valle, CP violation and neutrino oscillations. Prog. Particle Nuclear Phys. 60(2), 338-402 (2008) 